\title{
COMPARISON OF PIXEL AND OBJECT-BASED CLASSIFICATION TECHNIQUES FOR GLACIER FACIES EXTRACTION
}

\author{
S. D. Jawak ${ }^{1,2}$, S. F. Wankhede $2,3 *$, A. J. Luis ${ }^{2}$ \\ ${ }^{1}$ Svalbard Integrated Arctic Earth Observing System (SIOS), SIOS Knowledge Centre, University Centre in Svalbard (UNIS), P.O. \\ Box 156, N-9171, Longyearbyen, Svalbard, Norway; shridhar.jawak@gmail.com \\ ${ }^{2}$ Earth System Science Organization - National Centre for Antarctic and Ocean Research, Ministry of Earth Sciences, Government of \\ India, Headland Sada, Vasco-da -Gama, Goa 403804, India; alvluis@ ncaor.gov.in \\ ${ }^{3}$ Department of Geoinformatics, Mangalore University, Mangalore, Karnataka 574199, India; swankhede436@ gmail.com
}

Commission V, SS: Atmosphere, Ocean, Weather and Climate

KEY WORDS: Glacier facies, object-based methods, pixel-based methods, WorldView-2, customized spectral indices.

\begin{abstract}
:
Glacier facies are zones of snow on a glacier that have certain specific spectral characteristics that enable their characterization. The accuracy of their extraction will determine the end accuracy of the distributed mass balance model calibrated by this information. Therefore, coarse to medium resolution satellites are not preferable for this particular function as the data derived from such sensors will potentially blur out the minute spatial variations on the surface of a glacier. Very high resolution (VHR) sensors (such as, WorldView (WV)-1, 2, 3) are thus much more suited for this particular task. Hence, this study aims to extract the available glacier facies on the Sutri Dhaka glacier, Himalayas, using very high-resolution WorldView-2 (WV-2) imagery. Extensive pre-processing of the imagery was performed to prepare the data for this purpose. The steps incorporated for this purpose consist of 1) Data Calibration, 2) Mosaicking, 3) Pan Sharpening, 4) Generation of 3D surface, and 5) Digitization. Using image classification as the primary method of information extraction, this study tests the ever-popular pixel-based classification technique against the uprising object-based classification technique. In doing so, this study aims to determine the most accurate technique of information extraction for the WV -2 imagery in the given scenario. The presence of unique bands (Coastal $(0.40-0.45 \mu \mathrm{m})$, Red Edge $(0.705-0.745 \mu \mathrm{m})$, NIR-1 (0.770$0.895 \mu \mathrm{m})$ and NIR-2 $(0.86-1.04 \mu \mathrm{m})$ in the multispectral range of WV-2, allows this study to perform facies classification through the development of customized spectral index ratios (SIRs) in the object-based domain. Establishment of thresholds was hence necessitated for information extraction through the developed SIRs. Three supervised classifiers, namely, a) Mahalanobis distance, b) Maximum likelihood, and c) Minimum distance to mean, were then used to perform classification, thereby allowing a comparative analysis between the classification schemes. Accuracy assessment for each classification scheme was performed using error matrices. The object-based approach achieved an overall accuracy of $90 \%(\kappa=0.88)$ and the highest overall accuracy among the pixel-based classification methods is $78.57 \%(\kappa=0.75)$. The results clearly portray that the object-based method delivered much higher accuracy than the pixel-based methods. The carry home message is that future studies must examine the transferability and accuracy of the customized SIRs in varying scenarios, as different scenarios will require varying threshold adjustments. Forthcoming studies can also develop sensor specific and unique indices for other sensors that are suitable for such applications.
\end{abstract}

\section{INTRODUCTION}

Glaciers consist of various zones that are primarily differentiated based on specific characteristics. These characteristics are based on variables, which include, but are not limited to, moisture, texture, hardness, impurities, stratification, grain size, etc. These characteristics themselves are continuously transforming due to changing meteorological conditions (Benson, 1962). The two predominant zonations of a glacier are the accumulation zone and the ablation zone. The line of transition between them is called the Equilibrium Line Altitude (ELA). These two zones are a concatenation of several small zones having discernible superficial expressions due to changing circumstances. These are referred to as glacier facies (Hall et al., 1988). Glacier facies are superficial manifestations of the interplay of snow, the climate and the surrounding geology. Each facies occurs as snow metamorphoses through the glacier. Owing to seasonal changes, these glacier facies vary in spatial distribution and quantity throughout the year. As the properties of each facies vary, so does the impact of each vary on the overall health of the glacier. Therefore, although glacier facies are a resultant feature of snow interactions, they are distinct from the erosional and depositional features produced by the flow of the glacier (Benn and Evans, 2014). Ali et al., (2017), assessed the effects of changing glacier facies on the dimensions of the glacier through recession, deglaciation and meteorological parameters. Thus, it can be inferred that these superficial expressions (facies) are visual cues of the current health of the glacier (Jawak et al., 2018).

Mass balance modelling is the mechanism by which the changes experienced by a glacier are quantified. Several types of models have been developed (Jóhannesson et al., 1995; Hock, 1999; Klok and Oerlemens, 2002; and Braun and Hock, 2004) to perform single dimensional (ablation) as well as spatially distributed (ablation and energy balance) modelling. Braun et al., (2007), attempted to fill out the void sites of distributed mass balance models by deriving glacier facies data from Landsat satellite images on the Engabreen glacier in northern Norway. These void sites are a result of few on site data points that often leave out vast extents of the glaciers. Thus, it can be concluded that as each facies has a differential effect on the melting/recession of the glacier (Ali et al., 2017) the accurate 
extraction and analysis of glacier facies would inadvertently lead to much more accurate distributed mass balance modelling.

The beginnings of such complex modelling, centres around the accurate extraction of glacier facies. Image classification is a widely accepted form of information extraction from remotely sensed data. Coarse to medium resolution satellite datasets deliver good classification accuracies using conventional pixel based classification, however, high resolution and even very high resolution (VHR) imageries do not yield good classification accuracies through the same methods. This is attributed to the resultant salt and pepper/speckled effect of very high resolution classification (Gao and Mas, 2008). To overcome this, the utilization of object based classification has been generating momentum for land cover mapping through very high resolution data (Yadav et al., 2015; Weih and Riggan, 2010). Although such studies have been performed over land cover, they have not been tested exhaustively over glaciated regions. Therefore, this study yearns to map the available range of glacier facies of the Sutri Dhaka glacier, Himalayas. This mapping has been carried out using both pixel and object based classification (OBC) techniques. Thus, creating a comparative platform for the assessment of classification accuracies. This comparison was performed using error matrices.

\section{STUDY AREA AND DATA}

The present study area is the Sutri Dhaka glacier. This glacier lies in the upper Chandra basin of the western Himalaya (Figure 1), in the western part of the Lahaul-Spiti valley of Himachal Pradesh. The total area of the extracted glacier in this study is $24.93 \mathrm{~km}^{2}$. The extraction of accurate facies through unique methods necessitates unique selection of data. The data selected for this study is the WorldView-2 (WV-2) VHR data. The eight bands offered by WV-2 address issues such as insufficient feature details and limited analysis techniques (Jawak and Luis, 2013).

\begin{tabular}{|c|c|c|}
\hline \multicolumn{2}{|c|}{ Data } & Date of Acquisition \\
\hline \multirow{2}{*}{ WorldView-2 } & Panchromatic & 16 October 2014 \\
\hline & Multispectral & 16 October 2014 \\
\hline \multicolumn{2}{|c|}{ Aster GDEM v2 } & 16 January 2017 \\
\hline
\end{tabular}

Table 1. Data utilized

As described in Table 1, an Aster GDEM v2 (30m) was used in addition to the WV-2 image, for precise delineation through active reference from a generated 3-dimensional surface. This study attempts to map the available glacier facies using WV-2 VHR data (Table 2) by devising customized spectral index ratios (SIRs) within an object based sphere. This classification is then tested against pixel based supervised classification for an evaluation of the accuracy.

\begin{tabular}{|ccc|}
\hline WV-2 Bands & $\begin{array}{c}\text { Wavelength Range } \\
(\boldsymbol{\mu m})\end{array}$ & $\begin{array}{c}\text { Pixel Size } \\
(\mathbf{m})\end{array}$ \\
\hline PAN & $0.45-0.80$ & 0.5 \\
\hline Coastal & $0.40-0.45$ & 2 \\
\hline Blue & $0.45-0.51$ & 2 \\
\hline Green & $0.51-0.58$ & 2 \\
\hline Yellow & $0.565-0.625$ & 2 \\
\hline Red & $0.63-0.69$ & 2 \\
\hline
\end{tabular}

\begin{tabular}{|ccc|}
\hline Red Edge & $0.705-0.745$ & 2 \\
\hline NIR-1 & $0.770-0.895$ & 2 \\
\hline NIR-2 & $0.86-1.04$ & 2 \\
\hline
\end{tabular}

Table 2. WorldView-2 sensor specifications

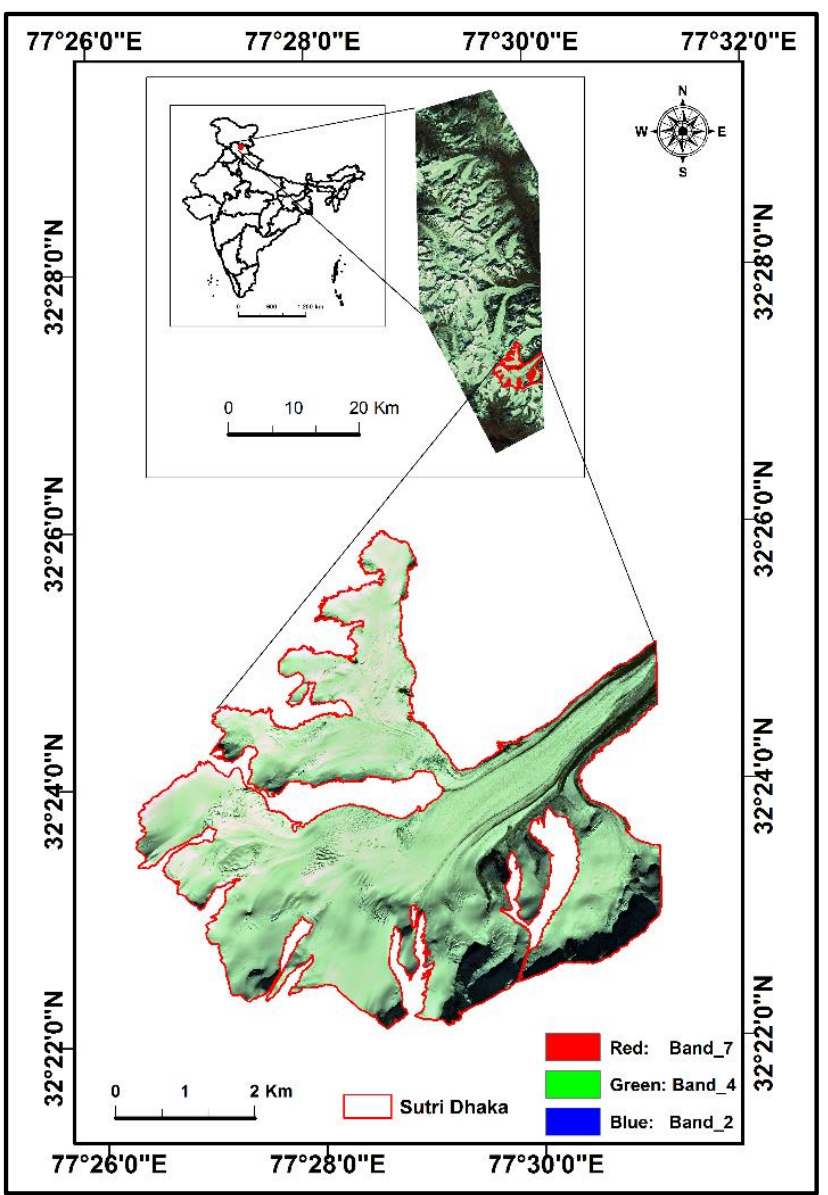

Figure 1. Geographical location of the study area.

\section{METHODOLOGY}

The methodology used in this study can be separated into a sequence of blocks; block a.) Pre-processing; block b.) Pixel based classification, block c.) Object based classification; and block d.) Accuracy assessment (Figure 2).

\subsection{Pre-processing}

The derivation of information from multispectral data requires a correction of the disturbances to the signal (Deshayes et al., 2006). Incorporating the procedure described by Jawak et al., 2017, pre-processing was performed to minimise the atmospheric effects over the data. The pre-processing block consisted of several sub blocks such as; (1) Data Calibration; (2) Mosaicking; (3) Pan sharpening; (4) Generation of 3D surface; (5) Extraction of study area. The raw data (courtesy of DigitalGlobe) comprised of 21 individual tiles, which were originally geometrically corrected to the projection system of UTM WGS $8443 \mathrm{~N}$ and the geographic co-ordinate system of GCS WGS 1984. 
Following Jawak and Luis, (2016); data calibration was performed by (a) The conversion of raw DN values to at sensor spectral radiance, followed by, (b) The conversion of at sensor radiance to at sensor or surface reflectance (FLAASH atmospheric correction, ENVI 5.3). Seamless mosaicking was performed to concatenate the tiles to assemble the final image of the study region. Pansharpening was performed using the GramSchmidt (GS) pan sharpening method (Laben and Brower, 2000) to enhance the spatial resolution of the multispectral WV-2 data (Figure 2). Extraction of the study glacier was performed by delineation of the glacier boundary with assistance from a 3dimensional draped surface generated by an Aster GDEM v2. The delineated boundary was utilized to extract the study glacier.

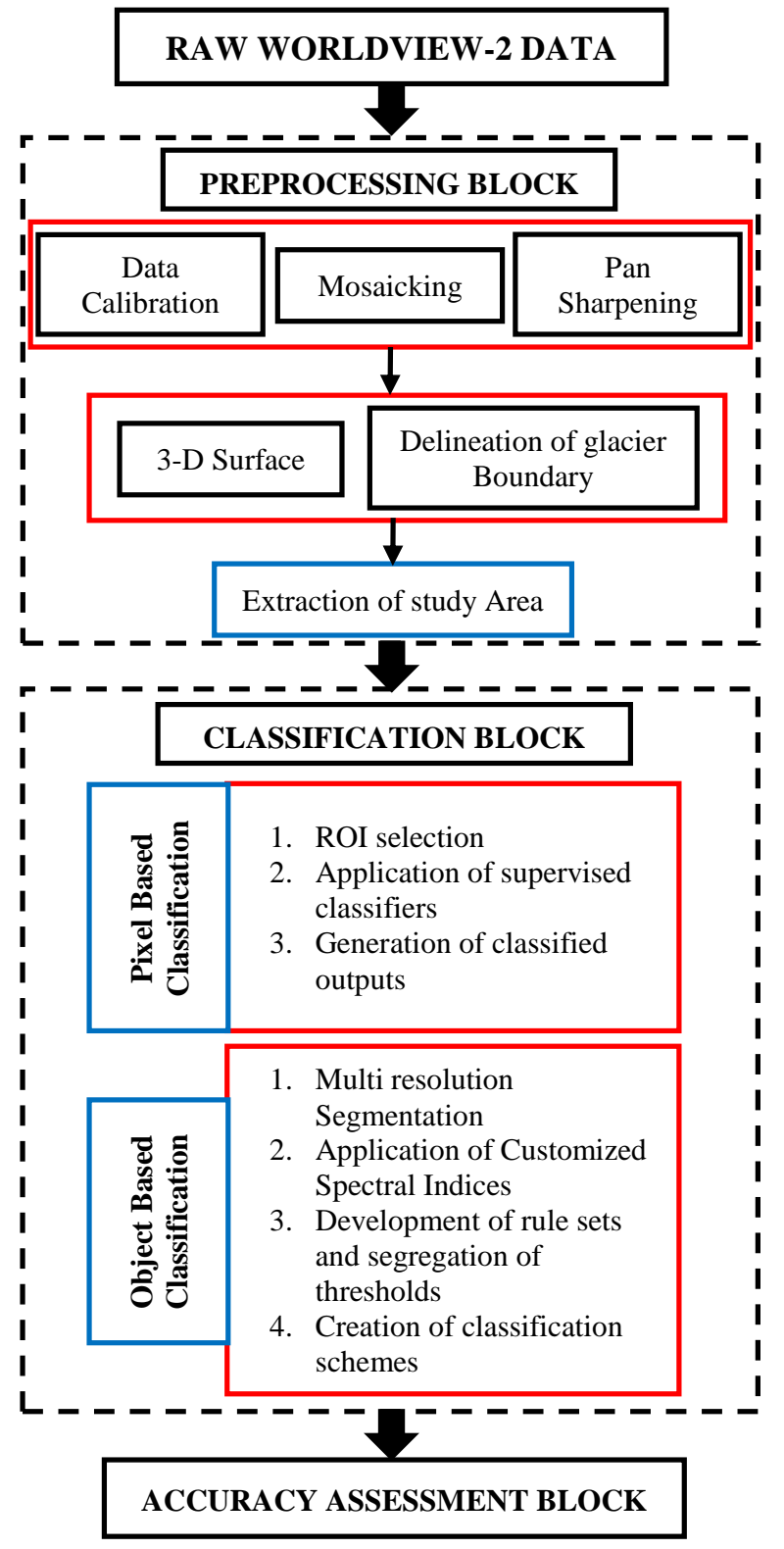

Figure 2. Protocol devised for the study

\subsection{Pixel Based Classification}

This study performed pixel based classification through the supervised classification technique. Three supervised classifiers were employed in this study, viz., (1) Mahalanobis Distance (MHD); (2) Maximum Likelihood (MXL); and (3) Minimum Distance (MD). This classification was performed using the TERCAT (Terrain Categorization) tool under the SPEAR (Spectral Processing Exploitation and Analysis Resource) automated workflow in ENVI 5.3. This tool provides for the generation of outputs from multiple classification algorithms via a single input. This input is provided through the means of regions of interests (ROIs) selected from analysis of spectral plots of the concerned targets. Classification in this technique is performed by first selecting ROIs, applying the supervised classifier and subsequently generating the output.

\subsection{Object Based Classification}

Classification in the object-based domain was performed using eCognition Developer 64. The first step in this process is multiresolution segmentation. This particular segmentation algorithm was selected as it increases the overall homogeneity of the created objects (Definiens Developer, 2012). The objects created as a result of segmentation obtain their characteristics from user-defined parameters such as image layer weights, scale parameter, shape and compactness. Layer weights were assigned as follows, NIR1=4, NIR2=3, Coastal=2, Green=2, Red Edge=2, Blue $=1$, Yellow $=1$, Red $=1$. The scale was set at 300, the shape at 0.4 and the compactness was set at 0.8 . Segmentation was then followed by the development of customized spectral index ratios (SIRs) to isolate objects having the desired spectral characteristics based on visual scrutiny and spectral curve analysis. The bands used to develop the customized SIRs were chosen after scrupulous attempts and exhaustive trials. Each band of the WV-2 sensor was tested in the form of various combinations to achieve isolation of facies. Three customized SIRs were developed (Table 3 ) after this rigorous procedure that enabled classification of the detectable facies.

Index no. Mathematical expression

\begin{tabular}{cc}
\hline SIR1 & $\frac{Y E L L O W}{\frac{N I R 1+N I R 2}{2}}$ \\
\hline SIR2 & $\frac{\frac{R E D E D G E}{N I R 1+N I R 2}}{2}$ \\
\hline SIR3 & $\frac{B L U E}{N I R 1+N I R 2}$ \\
& $\frac{N}{2}$
\end{tabular}

Table 3. The devised customized spectral index ratios

Engagement of indices entails the necessity of thresholds. Thus, as each threshold from one index or a combination of thresholds from one or multiple indices may be used to extract one particular facies, it would suffice to conclude that these thresholds are in fact unique specifications of the spectral characteristics of the resultant extracted facies (Jawak et al., 2017).

Shadowed areas in this image, owing to the unique conditions, are not entirely discernible using these SIRs. This is because shadowed areas vary spectrally within themselves as well as between each other. Therefore, it was necessary to manually digitize shadowed regions in ArcGIS 10.3. A tiny portion of 
valley rock was also digitized by the same procedure. The facies thus extracted in total using both classification techniques were wet snow, glacier ice, ice mixed debris, thick debris, crevasses, shadow and valley rock.

\subsection{Accuracy Assessment}

Seventy random points were assigned equally among the seven classes by analysis of the spectral plots of image targets. These points were then utilized for determination of the accuracy through several measures calculated by error matrices (Lillesand et al., 2004). The measures were: (1) Error of commission (EC); (2) Error of omission (EO); (3) Producer's accuracy (PA); (4) User's accuracy (UA); (5) Overall accuracy (OA); and (6) Kappa statistic ( $\kappa)$.

\section{RESULTS AND DISCUSSION}

Classification was performed on the extracted study area. This implies that the spectral variations within the shadowed regions were present at the time of object based classification. This led to the overestimation of certain facies within the shadowed area. Masking out the shadowed area would lead to erroneous results in the area wise distribution of the classified facies. Hence, it was imperative to erase the shadowed regions from the classified outputs using the Erase tool in ArcGIS 10.3. The final outputs therefore included no overestimations. Valley rock displayed similar spectral mixing and was subjected to the same procedure. Crevasse detection was highly promising with the SIR1. A combination of adjusted thresholds from SIR2 and SIR1 were necessary to efficiently extract wet snow and glacier ice. The distinction between thick and ice mixed debris were detected using SIR3. Each SIR uses specific bands to formulate a threshold parameter for classification. Therefore, each of the facies extracted hold discernible characteristics within the spectral range of the utilized bands. Each facies extracted is unique to its threshold range. This implies that the spectral response pattern of each of the facies is most influenced by the utilization of these SIRs. Thus, the three devised SIRs are the most influential with respect to the spectral response pattern of the target facies. The pixel based classification outputs required no such adjustments and corrections.

Comparative analysis purely based on measures of accuracy derived from the error matrices revealed that the object based classification did not only perform better than the pixel based classification, but far surpassed it (Table 4).

\begin{tabular}{|c|c|c|c|c|}
\hline $\begin{array}{c}\text { Measures of } \\
\text { Accuracy }\end{array}$ & $\begin{array}{c}\text { OBC } \\
(\%)\end{array}$ & $\begin{array}{c}\text { MHD } \\
(\%)\end{array}$ & $\begin{array}{c}\text { MXL } \\
(\%)\end{array}$ & $\begin{array}{l}\text { MD } \\
(\%)\end{array}$ \\
\hline $\begin{array}{c}\text { Error of } \\
\text { Commission }\end{array}$ & 10.00 & 21.43 & 17.14 & 2.86 \\
\hline $\begin{array}{c}\text { Error of } \\
\text { Omission }\end{array}$ & 9.05 & 23.57 & 17.44 & 17.81 \\
\hline $\begin{array}{l}\text { Producer's } \\
\text { Accuracy }\end{array}$ & 90.95 & 77.59 & 82.47 & 85.71 \\
\hline $\begin{array}{c}\text { User's } \\
\text { Accuracy }\end{array}$ & 90.00 & 77.14 & 82.86 & 77.14 \\
\hline $\begin{array}{c}\text { Overall } \\
\text { Accuracy }\end{array}$ & 90.00 & 70.00 & 78.57 & 75.71 \\
\hline $\begin{array}{c}\text { Kappa } \\
\text { Statistic }\end{array}$ & 0.88 & 0.65 & 0.75 & 0.72 \\
\hline
\end{tabular}

Table 4. Measures of Accuracy
The MHD (Figure 3) performed below average for the purpose of this study and resulted in an overall accuracy of $70 \%$. It delivered the highest errors of commission $(21.43 \%)$ and omission (23.57\%). The user's accuracy for MHD was $77.14 \%$, which was the same as that for MD (Figure 4). The MXL (Figure 5) classifier performed the best among the pixel based classifiers, delivering an overall accuracy of $78.57 \%$ and a kappa value of 0.75 . The object based classification delivered the highest accuracy and lowest errors. The overall accuracy achieved by the OBC (Figure 6) was $90 \%$. The kappa value obtained by the OBC was 0.88 . The errors of inclusion and exclusion achieved by the OBC were $10 \%$ and $9.05 \%$ (Table 4 ) respectively. Thus, the OBC proved to be the most effective and accurate classification technique

\section{CONCLUSION}

This study engages very high resolution WV-2 satellite data in pixel based and object based realms to perform classification of glacier facies. Classification, whether pixel or object based will be dependent on the precision of the spectral response pattern of the target. The entire pre-processing protocol devised for this study was suitably designed to facilitate optimal spectral response detection. The 3-Dimensional surface generated through Aster GDEM v2 (30m) was profoundly useful in the delineation of the glaciers. A significant feature of this study is the non-dependence on short wave infra-red (SWIR) bands. The protocol utilized in this study effectively maps glacier facies without including SWIR bands in the developmental phase. The three customized SIRs were highly efficient in mapping the target facies. Although a certain amount of manual correction was necessary to obtain the final output, the OBC yielded far greater accuracy than the PBC. The customized SIRs developed in this study can be tested in varying scenarios to determine their transformational capacity. Upcoming research may focus on creating a number of readily available index ratios for multiple satellite products to effectively map glacier facies.

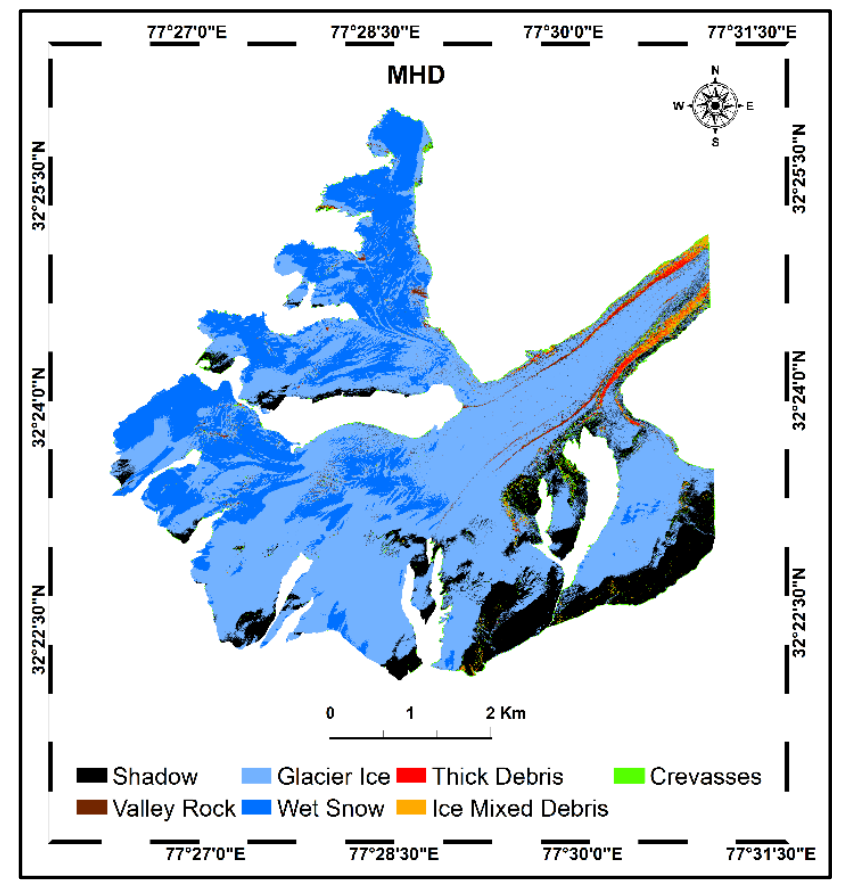

Figure 3. Mahalanobis distance classification map 


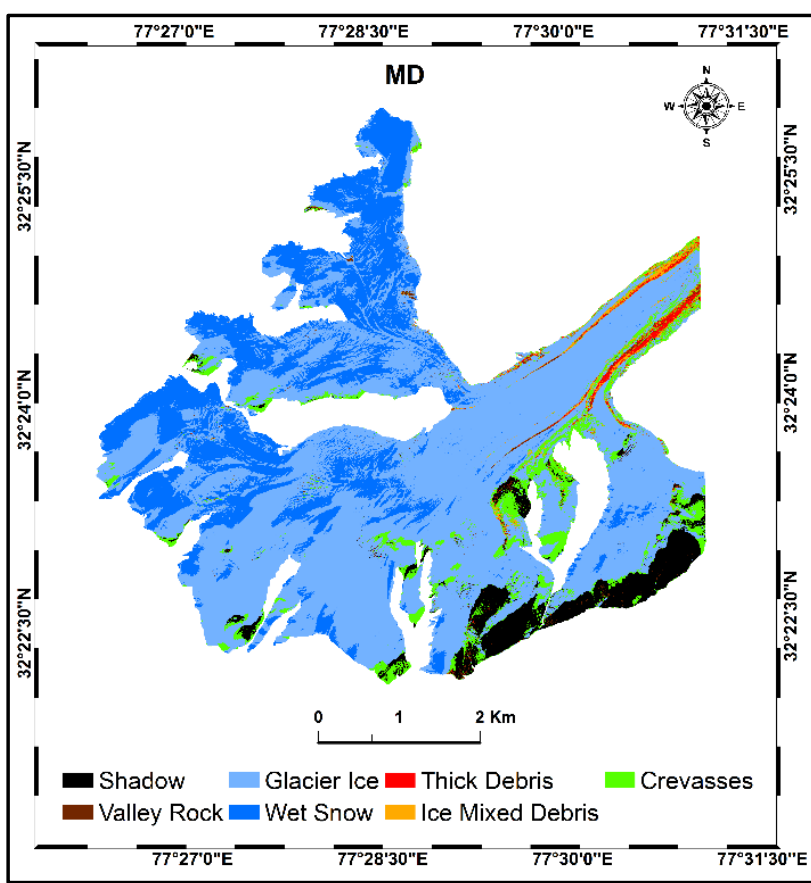

Figure 4. Minimum distance classification map

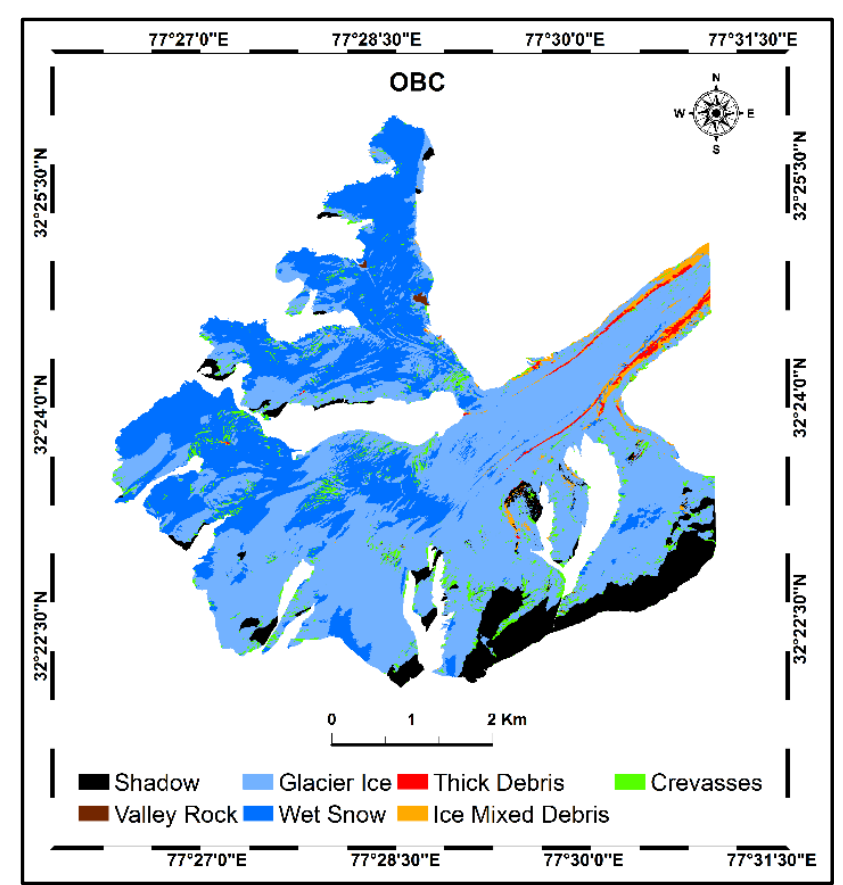

Figure 6. Object based classification map

\section{ACKNOWLEDGEMENTS}

The authors would like to thank DigitalGlobe for providing the imagery used in this study. We also acknowledge Dr. Rajan, former Director, ESSO-NCAOR and Dr. M. Ravichandran, Director, ESSO-NCAOR, for their encouragement and motivation for this research. The entire experiment, validation, manuscript preparation, and editing of the manuscript was conducted at the ESSO-NCAOR. The lead author (S.D.J) has recently moved to the SIOS, Longyearbyen, Norway.

\section{REFERENCES}

(Lisette) Klok, E. and Oerlemans, J., 2002. Model study of the spatial distribution of the energy and mass balance of Morteratschgletscher, Switzerland. Journal of Glaciology, 48(163), pp.505-518.

doi: https://doi.org/10.3189/172756502781831133

Ali, I., Shukla, A. and Romshoo, S., 2017. Assessing linkages between spatial facies changes and dimensional variations of glaciers in the upper Indus Basin, western Himalaya. Geomorphology, 284, pp.115-129.

doi: https://doi.org/10.1016/j.geomorph.2017.01.005

Benn, D. and Evans, D.J., 2014. Glaciers and glaciation. Routledge.

Benson, C. 1962. Stratigraphic studies in the snow and firn of the Greenland ice sheet, No. RR70. Cold Regions Research and Engineering Lab Hanover NH. http://acwc.sdp.sirsi.net/client/en_US/search/asset/1001392;jses sionid=351D596A6CE87F45BAEB04E7B9ECE897.enterprise15000 (accessed on 3 January 2018).

Braun, M., Schuler, T.V., Hock, R., Brown, I. and Jackson, M., 2007. Comparison of remote sensing derived glacier facies maps with distributed mass balance modelling at Engabreen, northern 
Norway. IAHS Publications-Series of Proceedings and Reports, 318, pp.126-134.

http://www2.gi.alaska.edu/ regine/Braun\&Schuler2007_IAHS. pdf

Definiens Developer, 2012. Ecognition Reference Book; Definiens AG, Bernhard-Wicki-Straße 5, 80636 München, Germany; pp. 34-37.

http://www.imperial.ac.uk/media/imperial-

college/medicine/facilities/film/Definiens-Developer-

Reference-Book-XD-2.0.4.pdf

Deshayes, M., Guyon, D., Jeanjean, H., Stach, N., Jolly, A. and Hagolle, O., 2006. The contribution of remote sensing to the assessment of drought effects in forest ecosystems. Annals of forest science, 63(6), pp.579-595.

Gao, Y. and Mas, J.F., 2008. A comparison of the performance of pixel-based and object-based classifications over images with various spatial resolutions. Online journal of earth sciences, 2(1), pp.27-35.

Hall, D., Chang, A. and Siddalingaiah, H., 1988. Reflectances of glaciers as calculated using Landsat-5 Thematic Mapper data. Remote Sensing of Environment, 25(3), pp.311-321. doi: https://doi.org/10.1016/0034-4257(88)90107-1

Hock, R., 1999. A distributed temperature-index ice- and snowmelt model including potential direct solar radiation. Journal of Glaciology, 45(149), pp.101-111.

doi: https://doi.org/10.1017/S0022143000003087

Jawak, S.D. and Luis, A.J., 2013. Very high-resolution satellite data for improved land cover extraction of Larsemann Hills, Eastern Antarctica. Journal of Applied Remote Sensing, 7(1), p. 073460

Jawak, S.D.; Luis, A.J., 2016. Geospatial mapping of Antarctic coastal oasis using geographic object-based image analysis and high resolution satellite imagery. In Proceedings of the International Society for Optics and Photonics, SPIE AsiaPacific Remote Sensing; pp. 98801Q-98801Q,

doi:10.1117/12.2222767

Jawak, S.D.; Wankhede, S.F.; Luis, A.J., 2017. Prospective of high resolution worldview-2 satellite data for geospatial surface facies mapping of an alpine glacier. In: Proceedings of the Asian Conference on Remote Sensing, New Delhi, India.

https://www.dropbox.com/sh/z0pws7ul29n7v1kd/AACaV9rFN cF01xNGnWwwgmF_a/177.pdf?dl=0

Jawak, S.D.; Wankhede, S.F.; Luis, A.J., 2018. Exploration of Glacier Surface FaciesMapping Techniques Using Very High Resolution Worldview-2 Satellite Data. Proceedings, 2 (7), 339. doi: https://doi.org/10.3390/ecrs-2-05152

Jóhannesson, T., Sigurdsson, O., Laumann, T. and Kennett, M. 1995. Degree-day glacier mass-balance modelling with applications to glaciers in Iceland, Norway and Greenland. Journal of Glaciology, 41(138), pp.345-358. doi: https://doi.org/10.3189/S0022143000016221

Laben, C.A. and Brower, B.V., Eastman Kodak Company, 2000. Process for enhancing the spatial resolution of multispectral imagery using pan-sharpening. U.S. Patent $6,011,875$.
Lillesand, T.M., Kiefer, R.W., Chipman, J.W., 2004. Remote sensing and image interpretation. John Wiley \& Sons, NJ, USA. Lu, D., Weng, Q., Moran, E., Li, G. and Hetrick, S., 2011. Remote sensing image classification. CRC Press/Taylor and Francis: Boca Raton, FL, USA, pp. 219-240.

Weih, R.C. and Riggan, N.D., 2010. Object-based classification vs. pixel-based classification: Comparative importance of multiresolution imagery. The International Archives of the Photogrammetry, Remote Sensing and Spatial Information Sciences, 38(4), p.C7.

Yadav, S., Rizvi, I. and Kadam, S., 2015. Comparative Study Of Object Based Image Analysis On High Resolution Satellite Images For Urban Development. International Journal of Technical Research And Applications. Special, (31), pp.105-110. 